\title{
Conversation entre les Muses, sous la direction de Lise Sabourin
}

\section{Alain Génetiot}

\section{(2) OpenEdition}

1 Journals

\section{Édition électronique}

URL : http://journals.openedition.org/studifrancesi/9716

DOI : 10.4000/studifrancesi.9716

ISSN : 2421-5856

Éditeur

Rosenberg \& Sellier

\section{Édition imprimée}

Date de publication : 1 décembre 2007

Pagination : 709-710

ISSN : 0039-2944

\section{Référence électronique}

Alain Génetiot, «Conversation entre les Muses, sous la direction de Lise Sabourin », Studi Francesi [En ligne], 153 (LI | III) | 2007, mis en ligne le 30 novembre 2015, consulté le 13 janvier 2021. URL : http:// journals.openedition.org/studifrancesi/9716; DOI : https://doi.org/10.4000/studifrancesi.9716

Ce document a été généré automatiquement le 13 janvier 2021.

\section{(c) (i) (2)}

Studi Francesi è distribuita con Licenza Creative Commons Attribuzione - Non commerciale - Non opere derivate 4.0 Internazionale. 


\title{
Conversation entre les Muses, sous la direction de Lise Sabourin
}

\author{
Alain Génetiot
}

\section{RÉFÉRENCE}

AA. VV., Conversation entre les Muses, sous la direction de Lise SABOURIN, Presses

Universitaires de Nancy, 2007 («Collection du Centre d'Étude des Milieux littéraires»),

pp. 248.

1 Lise Sabourin, directeur du Centre d'Étude des Milieux littéraires et artistiques (CEMLA) de l'Université Nancy 2, rassemble seize études concernant les influences réciproques entre les arts, du $\mathrm{XVI}^{\mathrm{e}}$ siècle à nos jours, et la réception par les artistes des œuvres ressortissant à un autre art que le leur (littérature, peinture, sculpture, musique, cinéma, mise en scène). La belle métaphore de la conversation, qui présuppose l'égale dignité des arts et l'amitié des créateurs, organise le recueil en un dialogue ouvert où se tisse un réseau de thèmes communs selon les arts envisagés dans une série de croisements réciproques. Elle pose en filigrane la question majeure de l'orphisme et de l'union des arts, qui, si elle reçoit ici avant tout une réponse à partir du XVIII siècle, pourrait être prolongée, moyennant l'adaptation des présupposés théoriques, aux siècles antérieurs. Conformément au principe de la collection, l'ouvrage est complété par un utile index nominum.

2 Dans l'ordre chronologique, Cécile HUCHARD (pp. 9-14) étudie, dans les Vrais Pourtraits des hommes illustres en piété et doctrine de Théodore de Bèze traduits par Simon Goulart (1581), le genre mixte du recueil de portraits orné d'emblèmes chrétiens: de manière paradoxale pour un recueil protestant, l'usage des images à fonction pathétique fait appel à la représentation corporelle pour donner à voir le corps de la République des lettres, dans une optique martyrologique qui affirme sa légitimité savante, conformément à l'idée humaniste de la conversation des grands hommes. Dans Les Costumes d'“Esther" ou le retour au désordre (pp. 15-22), Anne VERDIER et Didier DOUMERGUE 
analysent le succès paradoxal de la représentation d'Esther de Racine en 1689 à travers le code du costume. Alors que les demoiselles de Saint-Cyr jouaient les rôles d'hommes dans des robes à l'antique afin de ne pas choquer les bienséances, les costumes ont cependant été jugés dangereux, signe que le costume à lui seul suffit à transformer les jeunes filles, à leur corps défendant, en objet de spectacle.

Les contributions suivantes sont consacrées à l'époque des Lumières. Aurélie GÉRARD, avec Dom Calmet et les artistes de son temps (pp. 23-41), retrace les liens entretenus par le bibliste bénédictin avec les artistes à l'occasion de l'ornementation de ses ouvrages historiques et théologiques, mais aussi de la reconstruction et l'embellissement de ses abbayes, notamment celle lorraine de Senones, pour laquelle il collectionne tableaux, estampes, médailles et monnaies. Patricia MÉNISSIER (Voltaire et ses artistes: pour une union au service de l'image du philosophe?, pp. 43-56) examine l'iconographie de Voltaire au faite de sa célébrité mondaine par ses portraitistes et sculpteurs (Poncet, Huber, Pigalle, Denon, Houdon) pour poser la question de l'image du grand homme et de sa réception dans les milieux aristocratiques et littéraires qui font sa renommée. Dans Écriture picturale et morale: une rhétorique du discours pédagogique dans les romans féminins de la seconde moitié $d u$ XVIII ${ }^{e}$ siècle (pp. 57-74), Stéphanie MIECH montre comment les romancières (Mmes Riccoboni, Cottin, de Charrière, de Souza, de Genlis, de Krüdener, de Staël, de Duras) ont traduit dans leur écriture le motif pictural de la jeune fille exemplaire fourni par les peintres (Boucher, Chardin, Greuze, Lancret, Fragonard, Vigée-Lebrun, Boilly) pour tenir un discours moral sur l'idéal de bonheur domestique dans une perspective rousseauiste. Tatiana SIRoTcHouk étudie le développement des Lumières russes à partir de l'Académie de Kiev, qui a fourni à celle de Moscou les professeurs, traducteurs, journalistes qui ont permis la translatio studii de la culture européenne dans la Russie de Pierre $\mathrm{I}^{\mathrm{er}}$ et de la Grande Catherine (Les "Oiseaux migrateurs" ou la face inconnue des Lumières russes, pp. 75-91). À la même époque à Malte, les chevaliers d'origine française rédigent des descriptions des chefs-d'œuvre artistiques tandis que les voyageurs comme Vivant Denon, Patrick Brydone ou le comte de Borch évoquent les sites et les mœurs que Jean Houël, peintre du roi, représente dans son Voyage pittoresque des isles de Sicile, de Malte et de Lipari publié en 1782 (Carmen DEPASQUALE, Images littéraires et artistiques de Vile de Malte au XVIII siècle, pp. 93-106).

Avec le XIX ${ }^{e}$ siècle l'idée d'union des arts se fait plus centrale encore. Dans une inversion du point de vue, la peinture cherchant à représenter ce que la littérature définit comme «'irreprésentable: Médée furieuse de Delacroix (1838)» (pp. 107-117), Florence FIX, à partir de souvenirs littéraires - Euripide, mais aussi le Moïse en Egypte de Rossini et la Médée de Legouvé qui inspire un second tableau, aujourd'hui perdu - retrouve le rôle nécessaire pour le peintre de la memoria lettrée. Philippe ANDRÉS se tourne vers $L a$ Critique musicale de Banville, pp. 119-130, qui, de 1850 à 1881, commente les opéras de Gounod, Meyerbeer, Offenbach, Bizet, Wagner, avec une préférence à Berlioz, dans le souci d'observer l'aspect matériel des représentations et la volonté d'affirmer une conception orphique de l'union des arts. Laurence RICHER (Vivante sculpture: l'esthétique de Quinet, pp. 131-141), examine la figure du Créateur dans l'œuvre d'Edgar Quinet, d'Ahasverus et Prométhée à l'Essai d'une classification des arts, qui, à travers la figure du Démiurge sculpteur, définit un Art indispensable aux civilisations qu'il transcende. Wieslaw MALINOWSKI examine la Conjonction du littéraire et du pictural dans "Bruges-lamorte" de Rodenbach (pp. 143-157) en étudiant la pinacothèque rodenbachienne, qui va des peintres flamands aux préraphaélites, et l'utilisation poétique du clair-obscur qui 
donne au récit sa dimension crépusculaire, concluant que l'élément visuel nourrit tout le roman.

5 Enfin le $\mathrm{xx}^{\mathrm{e}}$ siècle témoigne d'une véritable symbiose entre les arts qui mettent leurs moyens en commun jusqu'à échanger leurs techniques. Ainsi dans Apollinaire et Delaunay: une amitié lumineuse (pp. 159-172), Stéphanie DEPOISSE présente la quête commune au poète et au peintre d'abstraction à travers l'orphisme. De même, à propos de Brancusi et ses amis écrivains (pp. 173-190), Mariana PERISANU met en évidence le dialogue du sculpteur avec les peintres, musiciens, poètes français Apollinaire, Tzara, Queneau, Morand, Fondane, Eliade, Cendrars, les Américains Ezra Pound, Joyce, et les Roumains de l'effervescence bucarestoise des années 1920-1930 Tudor Arghezi, Ion Vinea, Lucian Blaga, Ion Barbu. À partir de la même époque, mais avec deux siècles de distance, les écrivains français (de Supervielle et Cendrars à Dominique Fernandez et Michel Butor) retrouvent le baroque brésilien au XVIII ${ }^{e}$ siècle des églises du Minas Gerais (Régis tetTAmANZI, Aleijadinho, un sculpteur brésilien vu par les écrivains français, pp. 191-204). Mais l'apparition du septième art entraîne un dialogue entre le cinéma et la littérature que Matthieu RÉMY (pp. 205-218) examine chez des romanciers du second $\mathrm{xx}^{\mathrm{e}}$ siècle, qu'il s'agisse de réécriture par le cinéma pour Pérec, d'écrire d'après le cinéma pour Echenoz ou d'écrire comme le cinéma chez Modiano, dans un échange de techniques et de thèmes qui n'est pas toujours sans difficultés d'adaptation. Enfin Clara DEBARD (Esthétique contemporaine de la tragédie grecque, pp. 219-229) examine les récentes mises en scène du Prométhée enchaîné d'Eschyle par Stéphane Braunschweig, des pièces de Sophocle, Electre par Daniel Pierson, Ajax par Bérangère Jannelle, Antigone par Jacques Nichet, mais aussi d'Alceste d'Euripide et de la Médée de Sénèque par Jacques Lassalle. Ces relectures par des metteurs en scène contemporains mettent en évidence l'influence de la théorie d'Artaud tant sur le plan de la signification que dans l'organisation concrète du jeu et de l'espace scénique, mais appliquée aux chefsd'œuvre du passé, dans un retour à la "polyphonie des langages».

6 Par ses reprises et croisements thématiques, ce recueil est donc lui-même une conversation entre les Muses et un témoignage des nécessaires échanges et correspondances entre les artistes, avec l'idée sous-jacente que c'est à partir des réseaux amicaux de reconnaissance réciproque que se développe la création et la réception des œuvres, ce qui justifie le projet de la collection, l'analyse des milieux littéraires et artistiques. 